\title{
Diagnosis and successful visual biofeedback therapy using fiberoptic endoscopic evaluation of swallowing in a young adult patient with psychogenic dysphagia: a case report
}

\author{
Youngmo Kim ${ }^{1}$, Sang Hun Han ${ }^{1}$, Yong Beom Shin ${ }^{1,2}$, Jin A Yoon ${ }^{1,2}$, Sang Hun Kim ${ }^{1}$ \\ ${ }^{1}$ Department of Rehabilitation Medicine, Biomedical Research Institute, Pusan National University Hospital, Busan, Korea \\ ${ }^{2}$ Department of Rehabilitation Medicine, Pusan National University School of Medicine, Busan, Korea
}

Psychogenic dysphagia is a deglutition disorder characterized by a fear of swallowing, with no structural or functional causes. This report presents the case of a young male patient who had severe malnutrition due to psychogenic dysphagia and was provided visual biofeedback using fiberoptic endoscopic evaluation of swallowing (FEES). A healthy 25 -year-old man presented to our clinic with a complaint of throat discomfort when swallowing that had started 6 months prior. As the symptoms worsened, he became fearful of food spreading to his lungs after swallowing and the development of respiratory difficulties. His food intake gradually decreased, resulting in a weight loss of $20 \mathrm{~kg}$ within 2 months. Evaluation of organic and other functional causes of dysphagia was performed, but no abnormalities were detected. The sensation of a lump in his throat, fear of swallowing, and anxiety were transformed into somatic symptoms. The patient was diagnosed with psychogenic dysphagia. After visual biofeedback by a physician who performed FEES, the patient resumed eating normally and increased his food intake. If routine tests do not reveal structural or functional causes of dysphagia, assessment of a psychogenic swallowing disorder should be considered. FEES can help in the diagnosis and management of psychogenic dysphagia.

Keywords: Deglutition disorders; Globus sensation; Laryngoscopy; Somatoform disorders

\section{Introduction}

Psychogenic dysphagia is a swallowing disorder with no structural or organic cause. The etiology of psychogenic dysphagia is not well understood. The most common symptoms of the disorder include fear of swallowing, feeling lumps or fullness in the throat, and complaints of difficulty breathing when swallowing. These symptoms may be accompanied by somatic symptoms such as depression, anxiety, restlessness, insomnia, and anorexia $[1,2]$. Weight loss, avoidance of eating, and malnutrition can also occur, lowering the quality of life. Psychogenic dysphagia has been described using several terms, including choking phobia [3,4], globus hystericus [5], hysterical dysphagia [6], and phagophobia [7].

Although the prevalence of psychogenic dysphagia has not been well studied, one study indicated that $20 \%$ of patients with globus hystericus had psychogenic dysphagia [8], while another videoflu-

Received: October 11, 2021 • Revised: November 29, 2021 • Accepted: December 4, 2021

Corresponding author: Sang Hun Kim, MD, PhD

Department of Rehabilitation Medicine, Biomedical Research Institute, Pusan National University Hospital, 179 Gudeok-ro, Seo-gu, Busan 49241, Korea

Tel: +82-51-240-7485• Fax: +82-51-247-7485•E-mail: kel5504@gmail.com

Copyright (C) 2023 Yeungnam University College of Medicine, Yeungnam University Institute of Medical Science

This is an Open Access article distributed under the terms of the Creative Commons Attribution Non-Commercial License (http://creativecommons.org/licenses/by-nc/4.0/) which permits unrestricted non-commercial use, distribution, and reproduction in any medium, provided the original work is properly cited. 
oroscopic swallowing study (VFSS) showed that 22 out of 1,844 patients suffering from this condition [9]. Based on these cases, we can deduce that psychogenic dysphagia is often overlooked and is relatively common.

In this report, we present the details of a case of diagnosis and visual biofeedback intervention using fiberoptic endoscopic evaluation of swallowing (FEES) in a young male patient who suffered severe malnutrition and weight loss due to psychogenic dysphagia.

\section{Case}

Ethical statements: This study was approved by the Institutional Review Board (IRB) of Pusan National University Hospital (IRB No: 2111-022-109). Written informed consent from the patient was waived by the IRB.

A 25-year-old man visited our clinic with complaints of dysphagia that had started 6 months earlier. At that time, the patient was suspected of having respiratory symptoms related to coronavirus disease. To treat these symptoms, he was administered a single pill of the antimalarial drug combination pyronaridine-artesunate. Following treatment, the patient felt discomfort on the left side of his throat when swallowing. As time passed, the discomfort spread to the right side of his throat and was accompanied by pain. The symptoms worsened, causing breathing difficulties and a sensation of food spreading to his lungs. This caused the patient to become terrified of food intake. He had never experienced these symptoms previously and did not consult a psychiatrist.

He visited the Department of Pulmonology and Cardiology for assessment of dyspnea and chest discomfort. Blood tests, chest computed tomography, pulmonary function tests, and electrocardiography were performed, but no abnormalities were detected. His body weight markedly decreased from $85 \mathrm{~kg}$ (body mass index [BMI], $25.6 \mathrm{~kg} / \mathrm{m}^{2}$ ) to $66 \mathrm{~kg}$ (BMI, $19.9 \mathrm{~kg} / \mathrm{m}^{2}$ ) in 2 months. He was referred to the Department of Rehabilitation Medicine and was admitted for evaluation of dysphagia and nutritional management.

Physical and cranial nerve examinations did not reveal any abnormalities related to dysphagia. Laboratory findings were normal other than the presence of urine ketone $3+$, which is common in cases of starvation. Thyroid function tests and assessments for the levels of carcinoembryonic antigen and carbohydrate antigen 19-9 were performed to detect thyroid diseases or tumors. The results of the tests were within normal ranges. The patient's serum creatine kinase (CK) level was 114 U/L (range, 5-217 U/L), and his lysosomal acid $\alpha$-glucosidase activity was normal. A nerve conduc- tion study and electromyography were performed; however, no evidence of neuromuscular disease or peripheral neuropathy was observed. Additional brain evaluations were not performed because symptoms such as loss of balance, dysarthria, and diplopia were not present.

Imaging was performed to confirm whether a structural abnormality was the reason for the patient's swallowing disorder. Esophagography revealed no abnormal structure or esophageal dysmotility that could cause discomfort and pain in the throat (Fig. 1). A VFSS was performed, confirming a delay in the oral phase of swallowing solid food due to fear, but there were no abnormalities in bolus formation and pharyngeal contraction, nor was there premature spillage. Tracheal aspiration and upper esophageal sphincter impairment were not observed in the pharyngeal phase. FEES was also performed. The epiglottis movement was floppy, but movement of the vocal cords was normal, and there was no laryngeal structural pathophysiology (Fig. 2). During the swallowing tests, the patient's oxygen saturation and other vital signs were normal.

After admission, total parenteral nutrition was initiated for treatment of malnutrition and dehydration. He consumed only 260 $\mathrm{kcal}$ per day, which is approximately $11 \%$ of his daily nutritional requirements. He was diagnosed with severe malnutrition (marasmus). After providing sufficient nutrition to the patient, physical performance tests were implemented. The patient's handgrip strength was $25 \mathrm{~kg}$ (range, $37.7-57.5 \mathrm{~kg}$ ), and he was able to walk $462 \mathrm{~m}$ in a 6-minute walk test, which was $48.6 \%$ of the expected distance.

It was confirmed that there were no structural or functional

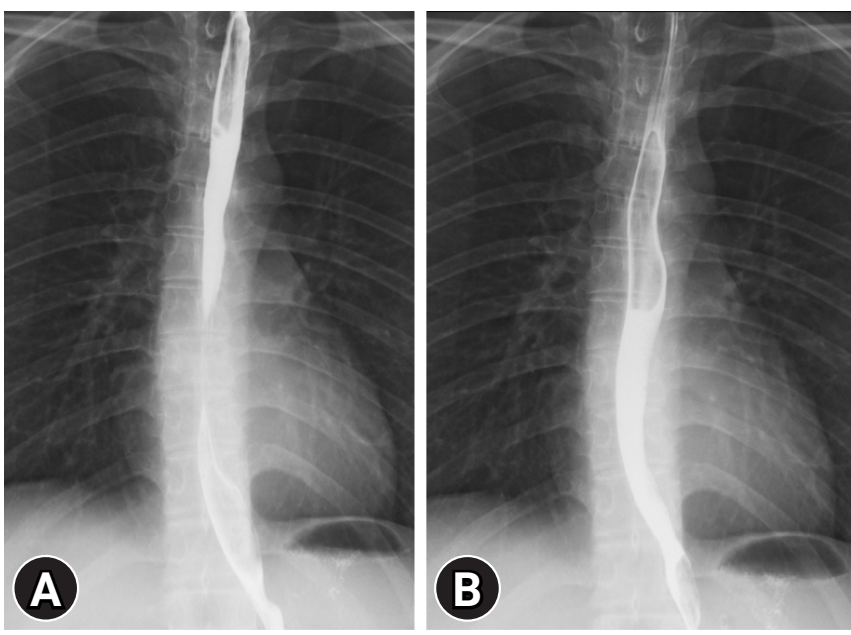

Fig. 1. Esophagography shows no structural abnormality or dysmotility from $(A)$ the upper and middle thoracic esophagus to (B) the lower thoracic esophagus and esophagogastric junction. 

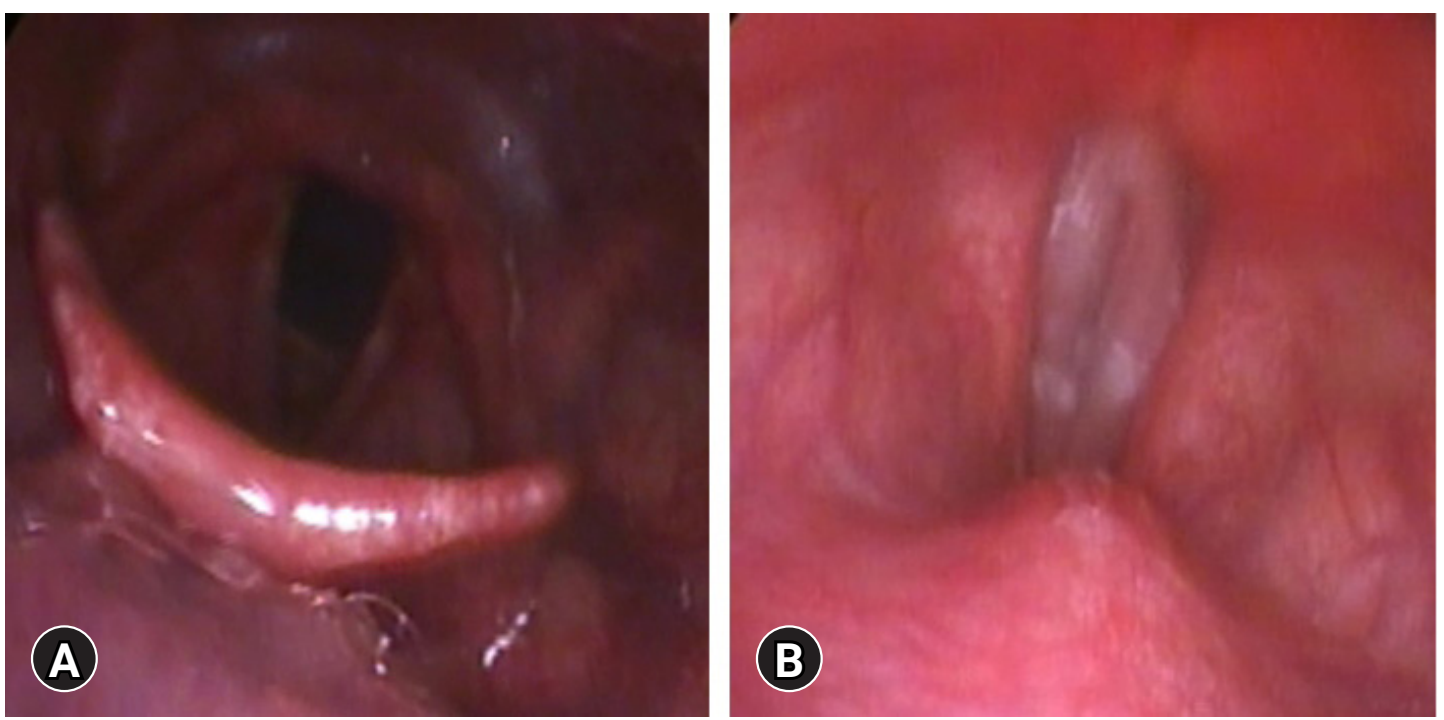

Fig. 2. Fiberoptic endoscopic evaluation of swallowing shows (A) no laryngeal penetration or aspiration and (B) no structural abnormality during phonation.

problems and that the patient's fear of swallowing and abnormal sensations were consistent with the pattern of psychogenic dysphagia, in which anxiety is transformed into somatic symptoms. He had previously taken numerous nutritional supplements owing to excessive health concerns. His family history revealed a younger sister with a diagnosis of schizophrenia. The patient was referred to the Department of Psychiatry where he was diagnosed with somatoform disorder. However, he did not want to undergo further psychiatric evaluations. After 1 week, a follow-up FEES was performed in a comfortable environment with a minimum number of observers. The test materials included an ionized beverage and porridge eaten at home instead of the hospital-provided diet of liquid and purée. No abnormalities related to symptoms were observed after the study. With guidance from a physician and with the help of images, the patient reconfirmed that tracheal aspiration in the airway did not occur. After the swallowing test, the patient was able to eat half of his meals and consume two cans of commercial enteral formulas twice a day (Harmonilan; Youngjin Pharm, Seoul, Korea). The patient gradually gained weight; after 3 months in the outpatient clinic, his body weight was $81 \mathrm{~kg}$ (BMI, $\left.24.4 \mathrm{~kg} / \mathrm{m}^{2}\right)$.

\section{Discussion}

In this report, we described a case of psychogenic dysphagia in a healthy young man. It is important to differentiate psychogenic dysphagia from organic or other types of functional dysphagia.

In psychogenic dysphagia, the fear of swallowing occurs due to experiencing or witnessing a choking event or feeling a lump or fullness in the throat when swallowing [1,3-5]. Therefore, obtaining a detailed and accurate medical history is important. The medical history should include when and under what circumstances dysphagia occurs and what symptoms are present. In this case, the patient complained of a lump and pain during swallowing and feared that food would spread to his lungs. The patient started experiencing dysphagia after taking a single antimalarial pill. Therefore, we considered the possibility of dysphagia and myopathy induced by the antimalarial drug. There have been reports of induced myopathy after taking antimalarial drugs (chloroquine and hydroxychloroquine) for at least 6 months $[10,11]$. However, there have been no reports of myopathy in people taking antimalarial drugs containing pyronaridine-artesunate.

We evaluated the patient for neuromuscular diseases that may cause swallowing disorders in healthy young men [12]. His serum CK levels were normal [13], and assessment of lysosomal acid $\alpha$-glucosidase activity for possible late-onset Pompe disease showed normal values [14].

The results of the nerve conduction study excluded neuromuscular disease. The patient's poor performance in the handgrip strength and 6-minute walk tests were believed to be due to transient general weakness caused by low food intake. As his nutritional intake increased, his physical performance gradually improved to $554 \mathrm{~m}$ in a follow-up 6-minute walk test. Brain lesions were excluded because there were no other neurological problems, apart from deterioration of physical function due to his poor general condition, and a cranial nerve examination was normal.

FEES and a VFSS were performed to determine the patient's swallowing ability. During the first swallowing test, unpleasant fa- 
cial expressions were noted, and effortful swallowing was observed at the start of the oral phase. There were no abnormal findings, and VFSS images confirmed that the food was not aspirated. Floppy epiglottis was observed in the patient's FEES. There was a reported case involving a patient with amyotrophic lateral sclerosis with floppy epiglottis [15]. Therefore, motor neuron disease was evaluated, but no related abnormalities were found. The patient attempted to eat after the swallowing test, but the feeling of aspiration persisted and he could not increase the amount of food he ate due to fear. Although he was initially diagnosed in the Department of Psychiatry, he refused further evaluation and intervention.

FEES is a treatment method that uses visual feedback and has several advantages. Anatomical structures are presented on a video screen, making it easy for patients to understand their swallowing physiology. Since there is no radiation exposure, patients can try swallowing training using visual feedback without time limitations. In addition, barium contrast agent is not required during the examination, allowing the patient to eat a usual meal with familiar tastes and flavors. Therefore, based on these advantages and the results of a previous study [16], visual biofeedback therapy using FEES was attempted. The follow-up FEES environment was made as comfortable as possible to minimize the psychological anxiety felt by the patient. The physician provided repeated explanations and reassurance, and the patient could see on the monitor that no aspiration was occurring during swallowing. The patient gradually overcame the fear of swallowing and began to eat. After observation at the outpatient clinic for more than 3 months, his food intake and body weight increased. A combination of psychological treatment and dysphagia therapy is the most effective treatment for psychogenic dysphagia [1]. The psychological treatment includes behavior therapy, insight-oriented therapy, and family therapy. The dysphagia therapy includes a swallowing exercise program and education on normal swallowing physiology. Reportedly, pharmacological treatment is also effective in treating psychogenic dysphagia [3]. However, if psychiatric evaluation and treatment are not possible for various reasons, visual biofeedback by the physician who performs FEES helps to improve the symptoms of psychogenic dysphagia. FEES visualizes the oropharyngeal phase of swallowing and reassures the patient. Therefore, FEES should be considered one of the best options for the diagnosis and management of psychogenic dysphagia. FEES has the advantage of providing visual biofeedback therapy and diagnosis simultaneously. Thus, it could be used as a treatment strategy for various swallowing disorders in the future.

\section{Notes}

\section{Conflicts of interest}

No potential conflict of interest relevant to this article was reported.

\section{Funding}

This work was supported by a clinical research grant from Pusan National University Hospital in 2021.

\section{Author contributions}

Conceptualization: YK, SHK, YBS, JAY; Data curation: SHK, SHH; Formal analysis: YK, SHK, SHH, YBS, JAY; Funding acquisition: SHK, YBS, JAY; Methodology, Project administration, Visualization, Investigation, Resources: YK, SHK; Supervision, Validation: SHK, YBS, JAY; Writing-original draft: YK; Writing-review \& editing: YK, SHK, YBS, JAY.

\section{ORCID}

Youngmo Kim, https://orcid.org/0000-0002-2518-530X

Sang Hun Han, https://orcid.org/0000-0002-0558-1399

Yong Beom Shin, https://orcid.org/0000-0001-5026-1696

Jin A Yoon, https://orcid.org/0000-0001-5762-0559

Sang Hun Kim, https://orcid.org/0000-0003-4849-5228

\section{References}

1. Bülow M. Psychogenic dysphagia. In: Shaker R, Belafsky PC, Postma GN, Easterling C, editors. Principles of deglutition. New York: Springer; 2013. p. 771-6.

2. Barofsky I, Fontaine KR. Do psychogenic dysphagia patients have an eating disorder? Dysphagia 1998;13:24-7.

3. Scemes S, Wielenska RC, Savoia MG, Bernik M. Choking phobia: full remission following behavior therapy. Braz J Psychiatry 2009;31:257-60.

4. McNally RJ. Choking phobia: a review of the literature. Compr Psychiatry 1994;35:83-9.

5. Finkenbine R, Miele VJ. Globus hystericus: a brief review. Gen Hosp Psychiatry 2004;26:78-82.

6. Ciyiltepe M, Türkbay T. Phagophobia: a case report. TurkJ Pediatr 2006;48:80-4.

7. Shapiro J, Franko DL, Gagne A. Phagophobia: a form of psychogenic dysphagia: a new entity. Ann Otol Rhinol Laryngol 1997; 106:286-90.

8. Ravich WJ, Wilson RS, Jones B, Donner MW. Psychogenic dysphagia and globus: reevaluation of 23 patients. Dysphagia 1989;4:35-8. 
9. Malcomson KG. Radiological findings in globus hystericus. Br J Radiol 1966;39:583-6.

10. Casado E, Gratacós J, Tolosa C, Martínez JM, Ojanguren I, Ariza A, et al. Antimalarial myopathy: an underdiagnosed complication?: prospective longitudinal study of 119 patients. Ann Rheum Dis 2006;65:385-90.

11. Kalajian AH, Callen JP. Myopathy induced by antimalarial agents: the relevance of screening muscle enzyme levels. Arch Dermatol 2009; 145:597-600.

12. Knuijt S, KalfJG, de Swart BJ, Drost G, Hendricks HT, Geurts AC, et al. Dysarthria and dysphagia are highly prevalent among various types of neuromuscular diseases. Disabil Rehabil 2014; 36:1285-9.

13. Somer H, Dubowitz V, Donner M. Creatine kinase isoenzymes in neuromuscular diseases. J Neurol Sci 1976;29:129-36.

14. Hobson-Webb LD, Jones HN, Kishnani PS. Oropharyngeal dysphagia may occur in late-onset Pompe disease, implicating bulbar muscle involvement. Neuromuscul Disord 2013;23: 319-23.

15. Ito K, Chitose H, Kobayashi A. Upper airway obstruction caused by a floppy epiglottis: report of two cases of amyotrophic lateral sclerosis (ALS). Nihon Jibiinkoka Gakkai Kaiho 2009; 112:660-4.

16. Thottam PJ, Silva RC, McLevy JD, Simons JP, Mehta DK. Use of fiberoptic endoscopic evaluation of swallowing (FEES) in the management of psychogenic dysphagia in children. Int J Pediatr Otorhinolaryngol 2015;79:108-10. 